\title{
Prevalencia de síndrome de burnout en anestesiología y cirugía ortopédica en un centro en Chile
}

\author{
Héctor Fabio Losada-Morales ${ }^{1}$, Paula Rosaisabel Astudillo-Díaz ${ }^{1}$ \\ Samuel Fernández-Carriba ${ }^{2,3}$ y Jimena Isabel Jara-Ibaceta ${ }^{1}$
}

\section{Burnout prevalence in anesthesiology and orthopedic surgery in a center in Chile}

\begin{abstract}
Aim: To assess levels of burnout, including emotional exhaustion, personal accomplishment and depersonalization, in the daily work of academic doctors in the Orthopedic Surgery Service and Central Surgery Service of the Hospital Hernán Henríquez Aravena (HHHA) and Surgery Department in the Universidad de La Frontera, Temuco, Chile. Materials and Method: Cross-sectional study. 48 subjects participated: 10 residents in orthopedic surgery, 8 residents in anesthesiology, 12 academic anesthesiologists and 18 academic orthopedic surgeons. Instruments applied under informed consent: Maslach Burnout Inventory (MBI). Analysis: Measures of central tendency and percentage, independent sample t-tests. Cronbach's alpha coefficients of the MBI. Results: The overall prevalence of burnout syndrome is $97 \%$, whereas by dimensions, emotional exhaustion showed a prevalence of $100 \%$, low personal accomplishment at work $100 \%$ and depersonalization $91.6 \%$. For orthopedic surgery by dimensions: emotional exhaustion showed a prevalence of $100 \%$, low personal accomplishment at work $100 \%$ and depersonalization $82 \%$. For anesthesiology: emotional exhaustion showed a prevalence of $75 \%$, low personal accomplishment at work $30 \%$ and depersonalization $30 \%$. Only gender and hours of sleep showed significant differences in emotional exhaustion, with higher scores for women and those who slept less than 7 hours. 0.7 Cronbach's alpha of the MBI. Conclusion: There is excessive emotional fatigue, low job felt accomplishment and depersonalization in orthopedic surgery residents and academic. In anesthesiology, emotional high exhaustion coexists with low personal accomplishment values and depersonalization high. In Chile, protection policies are mainly focused on Occupational Safety and Health with risk management, but there are no stress protection and mental health policies involving containment and support for the therapeutic work of medical professionals.
\end{abstract}

Key words: burnout professional; stress psychological; medical staff hospital; traumatology; anesthesiology.

\section{Resumen}

Objetivo: Evaluar el agotamiento emocional o cansancio emocional, realización personal y despersonalización en el quehacer de los médicos del Servicio de Traumatología y Pabellón Central del Hospital Hernán Henríquez Aravena (HHHA) y Departamento de Cirugía de la Universidad de La Frontera, Temuco, Chile. Materiales y Método: Corte transversal. 48 sujetos: 10 residentes de traumatología y 8 de anestesiología, 12 anestesiólogos y 18 traumatólogos académicos asistenciales. Se aplicó bajo consentimiento informado: Inventario de Maslash (MBI). Análisis: tendencia central, porcentaje, así como t de student y coeficientes de alfa de Cronbach. Resultados: La prevalencia global del síndrome de burnout es $97 \%$, por dimensiones, cansancio emocional mostró una prevalencia del 100\%; baja realización personal en el trabajo $100 \%$ y despersonalización $91,6 \%$. Para traumatología: cansancio emocional mostró prevalencia $100 \%$; baja realización personal en el trabajo $100 \%$, y despersonalización $82 \%$. Para anestesiología: cansancio emocional mostró prevalencia $75 \%$; baja realización personal en el trabajo $30 \%$ y despersonalización $30 \%$. Existen diferencias significativas en cansancio emocional siendo mayor en género femenino y en quienes duermen menos de $7 \mathrm{~h}$. El coeficiente de alpha de Cronbach del MBI fue de 0,7. Conclusión: Tanto residentes como académicos asistenciales de traumatología y anestesiología mostraron un elevado cansancio
Departamento de

Cirugía, Traumatología y Anestesiología, Universidad de La Frontera. Temuco, Chile. ${ }^{2}$ Marcus Autism Center en Children's Healthcare of Atlanta, USA.

${ }^{3}$ Emory University School of Medicine. Georgia, USA.

Recibido 2020-03-12 y aceptado 2020-08-03

Correspondencia a: Dra. Paula Rosaisabel Astudillo-Díaz paula.astudillo@ufrontera.cl 
emocional que coexiste con una baja realización personal en el trabajo y alta despersonalización. En Chile, las políticas de protección están principalmente enfocadas en la Seguridad y Salud Ocupacional con la gestión de los riesgos, pero no se observan políticas de protección al estrés y salud mental que involucren contención y apoyo a la labor terapéutica de los profesionales de la medicina.

Palabras clave: burnout profesional; estrés psicológico; personal médico hospitalario; traumatología; anestesiología.

\section{Introducción}

Desde la publicación de la teoría del síndrome general de adaptación por Hans Selye en $1950^{1}$, que describió el estrés como una respuesta trifásica incluyendo reacción de alarma, resistencia y agotamiento, muchos autores han investigado la respuesta de estrés en personal clínico. Por ejemplo, según Landa $^{2}$, las emociones presentes en la experiencia de estrés se pueden resumir como un estado de malestar psicológico marcado por la ansiedad, rabia, insatisfacción y el cansancio mantenido. De hecho, el principal elemento identificado es el estado de preocupación constante: un estado de ansiedad continuo, a la cual los trabajadores muchas veces no se logran adaptar dando paso al agotamiento emocional y al estrés.

Para entender los diferentes tipos de efectos negativos a la fase de agotamiento de la respuesta de estrés necesitamos describir el síndrome de burnout o síndrome de quemarse por el trabajo. La definición más aceptada ${ }^{3}$ es "el cansancio emocional que lleva a una pérdida de motivación y que suele progresar hacia sentimientos de inadecuación y fracaso". El síndrome de burnout se desarrolla de manera frecuente en profesionales asistenciales en las que el objeto de trabajo son otras personas (pacientes, familia, comunidad, otros profesionales) y se compone de tres dimensiones: a) agotamiento o cansancio emocional; b) despersonalización; c) la baja realización personal o logro. Maslach y Jackson ${ }^{4}$, lo definen como una respuesta inadecuada a un estrés emocional crónico cuyos rasgos principales son un agotamiento físico o psicológico y emocional, o pérdida de energía y fatiga, que puede manifestarse física, psicológicamente o como una combinación. Aparecen asimismo una serie de respuestas negativas hacia uno mismo como: moral baja, incremento de la irritabilidad, evitación de las relaciones profesionales, baja productividad, incapacidad para soportar la tensión, pérdida de la motivación hacia el trabajo y baja autoestima.

Una hipótesis respecto a la etiología del síndrome de burnout en profesionales de la salud en el con- texto de las organizaciones sanitarias, tiene que ver con que son profesionales que desarrollan su trabajo en contextos de relaciones sociales con otros profesionales en la institución, pacientes y sus familiares, y la coherencia e integración de estas relaciones con su estructura, sus políticas y procedimientos ${ }^{5,6}$. En otras palabras, son conflictos en las relaciones con pacientes y familiares, y limitada coherencia e integración de los sistemas, estructura, política y procedimientos de las organizaciones, que se pueden constituir en estresores y potenciadores del síndrome de burnout. Respecto a los efectos biológicos del estrés, pueden reflejarse en problemas cardiovasculares, musculoesqueléticos, de salud mental con capacidad reducida para regular emociones negativas, en crisis nerviosas, patologías gastrointestinales, heridas en el lugar de trabajo y funciones inmunes afectadas, entre otras ${ }^{7}$.

Al aproximarnos al contexto sociopolítico de la región de La Araucanía es la que presenta históricamente los indicadores biomédicos y sociales más deteriorados del país: alta ruralidad, años de vida potencialmente perdidos, y los índices de pobreza y pobreza extrema más altos del país, con un $27,9 \%$ y $10,6 \%$ respectivamente ${ }^{8,9}$. Estos elementos configuran las particularidades de los pacientes de la Región, a ello se agrega el rol de cada profesional como los anestesiólogos dedicados al alivio del dolor y al cuidado del paciente quirúrgico antes, durante y después del acto quirúrgico, también el soporte del paciente crítico en urgencias y la participación en cuidados paliativos ${ }^{10}$. El rol de la traumatología abarca más allá del campo de lesiones traumáticas; contempla también el estudio de patologías congénitas y cáncer. Otro aspecto a considerar es la creciente prevalencia de lesiones musculoesqueléticas asociadas a accidentes traumáticos, la práctica deportiva y la mayor esperanza de vida de nuestra población, por lo que se agrega una fuerte labor para lograr un retorno precoz a las actividades de la vida diaria de los pacientes. Por otra parte, tanto anestesiólogos como traumatólogos mantienen su rol en docencia e investigación.

Frente al panorama anterior, podemos esperar 
altos niveles del síndrome de burnout, así como los planteados en reciente estudio en cirujanos de la región ${ }^{11}$, es por ello la necesidad de evaluar la prevalencia del síndrome de burnout en el quehacer de los profesionales de la medicina de traumatología y anestesiología pertenecientes al Hospital Hernán Henríquez Aravena y al Departamento de Cirugía de la Universidad de La Frontera, Temuco, Chile, valorando los niveles de agotamiento emocional, realización personal y despersonalización en la dinámica de relaciones médicas asistenciales y académicas. Por otro lado, la exploración es un primer paso en torno a enfrentar el estrés en instituciones de salud de alta complejidad.

\section{Materiales y Método}

\section{Diseño}

Corte transversal.

\section{Sujetos}

48 personas participaron en el estudio, que incluyeron a residentes de traumatología y anestesiología, anestesiólogos y traumatólogos.

\section{Instrumento}

Maslach Bumout Inventory (MBI) el II semestre del año 2016 en su adaptación a la población española y validado en Latinoamérica ${ }^{12}$. El MBI plantea una serie de enunciados sobre sentimientos y pensamientos con relación a su interacción con el trabajo. Formado por 22 ítems que valoran con una escala tipo Likert, mediante un rango de 6 adjetivos que van de "nunca" a "diariamente", con qué frecuencia experimenta el sujeto cada una de las situaciones descritas en los ítems. Consta de tres subescalas: 1) Agotamiento Emocional o Cansancio Emocional (CE) formada por 9 ítems que se refieren a la disminución o pérdida de recursos emocionales o describen sentimientos de estar saturado y cansado emocionalmente por el trabajo; 2) Despersonalización (DE) formada por 5 ítems que describen una respuesta fría e impersonal y falta de sentimientos e insensibilidad hacia los sujetos objeto de atención;
3) Realización Personal en el trabajo (RP) compuesta por 8 ítems que describen sentimientos de competencia y eficacia en el trabajo; la tendencia a evaluar el propio trabajo de forma negativa y vivencia de insuficiencia profesional.

Se pidió informar de hábitos de sueño y datos sociodemográficos como: estado civil, número de hijos, experiencia laboral, años de experiencia en el lugar de trabajo.

\section{Aplicación del instrumento}

En agosto de 2016, en sala de reunión clínica, previo consentimiento informado. Se pidió expresamente que a cada uno de los 22 ítems del instrumento se respondiera expresando la frecuencia con que se tenía ese sentimiento, poniendo una cruz en la casilla correspondiente y el número que se considerara más adecuado. Tomó 15 minutos en total.

\section{Herramientas estadísticas}

Se construyeron bases de datos en el programa Acces $^{\circledR}$, Excel ${ }^{\circledR}$ y Stata 9.0 ${ }^{\circledR}$. Se realizó cálculos de medidas de tendencia central y porcentaje, así como $\mathrm{t}$-student para muestras independientes con un margen de seguridad de 95\%. Para evaluar los valores de los coeficientes de alfa de Cronbach, se utilizaron las recomendaciones de George y Mallery siendo: mayor a 0,7 aceptable, mayor a 0,6 cuestionable y mayor a 0,5 pobre.

Las puntuaciones de burnout se calificaron según la norma americana y la adaptación catalana, que determinan las categorías de bajo, medio y alto en cada una de las dimensiones según las puntuaciones (Tabla 1). Se considerará burnout cuando cualquiera de las tres dimensiones se encuentra en categorías media y alta. La prevalencia de burnout es el porcentaje de sujetos con puntaje medio y alto según el total de sujetos del grupo. Ahora, una baja realización personal muestra un puntaje que coincide con el nivel de identificación alto y sensación de logro con el nivel de identificación bajo.

\section{Consideraciones éticas}

El estudio fue aprobado por directivos del servicio de traumatología y pabellón central del HHHA,

Tabla 1. Puntuaciones y niveles de identificación de dimensiones de burnout

\begin{tabular}{|cccc|}
\hline Agotamiento emocional & Baja realización en el trabajo & Despersonalización & Nivel de identificación \\
Mayor o igual a 27 & Menor o igual a 30 & Mayor o igual a 14 & Alto \\
$17-26$ & $36-31$ & $9-13$ & Medio \\
$0-16$ & $48-37$ & $0-8$ & Bajo \\
\hline
\end{tabular}




\section{ARTíCULO ORIGINAL}

habiéndose verificado que cumplía con todas las consideraciones éticas de esta institución, incluyendo el consentimiento informado y anonimato de los participantes.

\section{Resultados}

Participaron 48 sujetos: 10 residentes de traumatología, 8 residentes de anestesiología, 12 anestesiólogos y 18 traumatólogos académicos asistenciales pertenecientes al Servicio de Traumatología y Pabelón Central del HHHA y Departamento de Cirugía, Traumatología y Anestesiología de la Universidad de La Frontera.

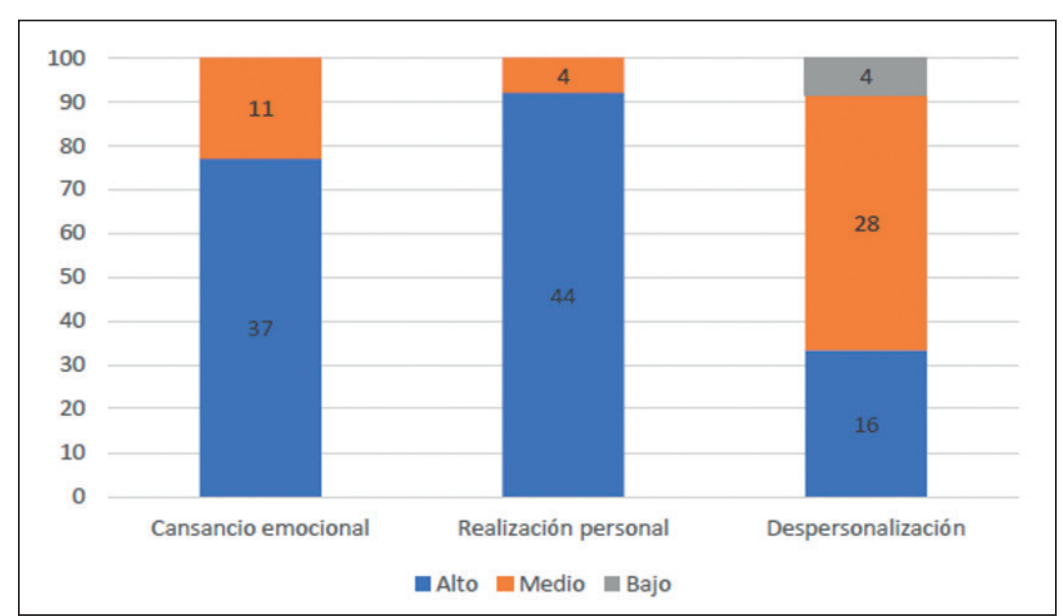

Figura 1. Número total de participantes que puntuaron en las categorías de alto, medio, bajo en cada una de las dimensiones $(n=48)$.

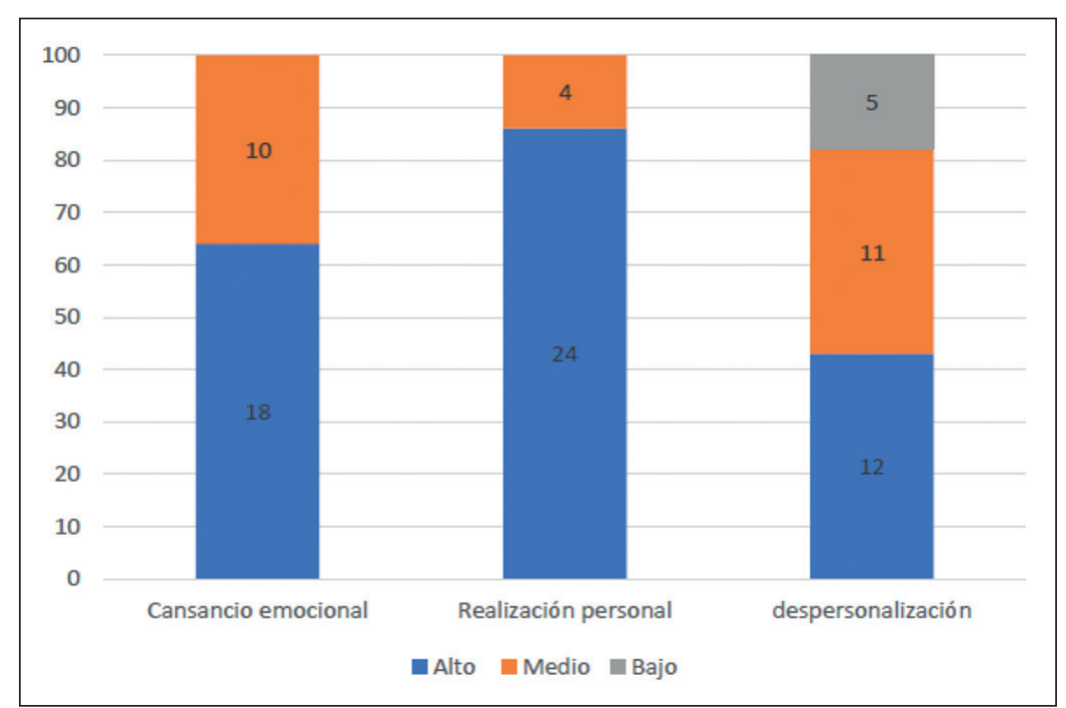

Figura 2. Número total de residentes y traumatólogos que puntuaron en las categorías alto, medio, bajo en cada subescala MBI $(n=28)$.
La consistencia interna de la escala de MBI, alpha de Cronbach de 0,7. En cada subescala, Agotamiento o Cansancio Emocional (CE) con valor de 0,88, Realización Personal (RP) 0,76 y Despersonalización (DE) un 0,61.

Como se aprecia en la Figura 1, la prevalencia global del síndrome en el total de participantes es $97 \%$. Cabe recordar que la prevalencia es el porcentaje de sujetos con puntaje medio y alto según el total de sujetos del grupo, es decir, en este caso son los 48 sujetos que respondieron la dimensión $\mathrm{CE}$, los mismos 48 sujetos la dimensión RP y posteriormente los mismos 48 responden la dimensión DE. Es por esta razón que se afirma que 140 sujetos (48 $\mathrm{x} 3$ ) puntuaron en alto y medio de 144 .

Por dimensiones, el cansancio emocional mostró una prevalencia de $100 \%$ (48 sujetos); baja realización personal en el trabajo 100\% (48 sujetos), y despersonalización 91,6\% (44 sujetos). En la Figura 1 se observa al número total de participantes que puntuaron en las diferentes categorías y dimensiones.

\section{Traumatólogos y residentes de traumatología}

Dentro del grupo de traumatología de 28 sujetos, el $35,7 \%$ residentes ( $60 \%$ de primer año) y $64,3 \%$ académicos asistenciales. El 83\% hombres. El promedio de edad $41 \pm 12,2$ años; el $66,6 \%$ casados y el $33,3 \%$ solteros. El 80\% con hijos (3 en promedio). El promedio de experiencia laboral $13 \pm 11$ años mientras que la experiencia en el lugar de trabajo un promedio de 8 con un mínimo de 0 a un máximo de 8 años. Respecto a hábitos de sueño, el $86,6 \%$ declaró dormir menos de $7 \mathrm{~h}$ diarias. Y ante la pregunta “siente estrés hoy?”, con una respuesta numérica de 1 a 10 siendo 10 la máxima expresión de estrés, declararon un promedio de $5 \pm 2,1$ con mínimo de 2 y máximo de 8.

Respecto a la escala de MBI, como se observa en la Figura 2, la prevalencia global del síndrome para el total de participantes de traumatología es $94 \%$, mientras que, por dimensiones, el cansancio emocional mostró una prevalencia de $100 \%$ (28 sujetos); baja realización personal en el trabajo 100\% y la despersonalización el 82\% (23 sujetos).

\section{Anestesiólogos/as y residentes de anestesiología}

En el grupo de anestesiología de 20 sujetos, el $40 \%$ residentes (50\% de primer año) y el $60 \%$ académicos asistenciales. El 35\% hombres, y el 65\% mujeres. El promedio de edad $38 \pm 9,4$ años; el 55\% estaban casados y el $40 \%$ sin pareja. El $75 \%$ con hijos ( 2 en promedio). El promedio de experiencia laboral de $11 \pm 8,4$ años mientras que la experiencia en el lugar de trabajo un promedio de 5 con un 
mínimo de 0 y máximo de 13 años. Un 75\% declaró dormir menos de $7 \mathrm{~h}$ y un $25 \%$ entre 7 y $8 \mathrm{~h}$. Ante la pregunta "¿siente estrés hoy?", declararon un promedio de $5 \pm 1,9$ con un mínimo de 1 y máximo de 8 .

Respecto a la escala de MBI, como se observa en la Figura 2, la prevalencia global del síndrome es $45 \%$ ( 9 sujetos), mientras que, por dimensiones, el cansancio emocional mostró una prevalencia del $75 \%$ ( 15 sujetos); baja realización personal en el trabajo 30\% (6 sujetos) y la despersonalización el 30\% (6 sujetos). En la Figura 3 se observa al número de participantes de traumatología que puntuaron en las diferentes categorías y dimensiones.

\section{Residentes y académicos}

A continuación se analizaron los grupos del total de residentes de traumatología y anestesiología $(\mathrm{n}=18)$ y académicos-asistenciales de traumatología y anestesiología $(\mathrm{n}=30)$, por separado.

\subsection{Total de residentes}

En la Figura 4 se observa al número de residentes que puntuaron en las diferentes categorías y dimensiones en la escala de MBI. La prevalencia global del síndrome para el total de residentes es 94\%, mientras que, por dimensiones, el cansancio emocional mostró una prevalencia del 100\% (18 sujetos); baja realización personal en el trabajo 100\% (18 sujetos) y la despersonalización el 83\% (15 sujetos).

\subsection{Total de académicos-asistenciales}

En la Figura 5 se observa al número de académicos asistenciales que puntuaron en las diferentes categorías y dimensiones, y la prevalencia global del síndrome para el total es $97,7 \%$, mientras que, por dimensiones, el cansancio emocional mostró una prevalencia del 100\% (30 sujetos); baja realización personal en el trabajo 100\% (30 sujetos) y la despersonalización el 93\% (28 sujetos).

\section{Variables demográficas y hábito de sueño del total de participantes}

Al explorar las variables sólo encontramos que existen diferencias significativas en el cansancio emocional siendo mayor en mujeres y en quienes duermen menos de $7 \mathrm{~h}$. En la Tabla 2 se muestra la comparación según sexo y horas de sueño según cada subescala de MBI.

Al observar la prevalencia según estos grupos, en mujeres fue del $94,8 \%$, mientras que el CE una prevalencia del 100\% (15 sujetos); una baja RP el $100 \%{ }^{15}$, y la DE el $93 \%$ (14 sujetos). En la Tabla 3 se muestra la puntuación del total de mujeres en las categorías alto, medio, bajo en cada subescala MBI.

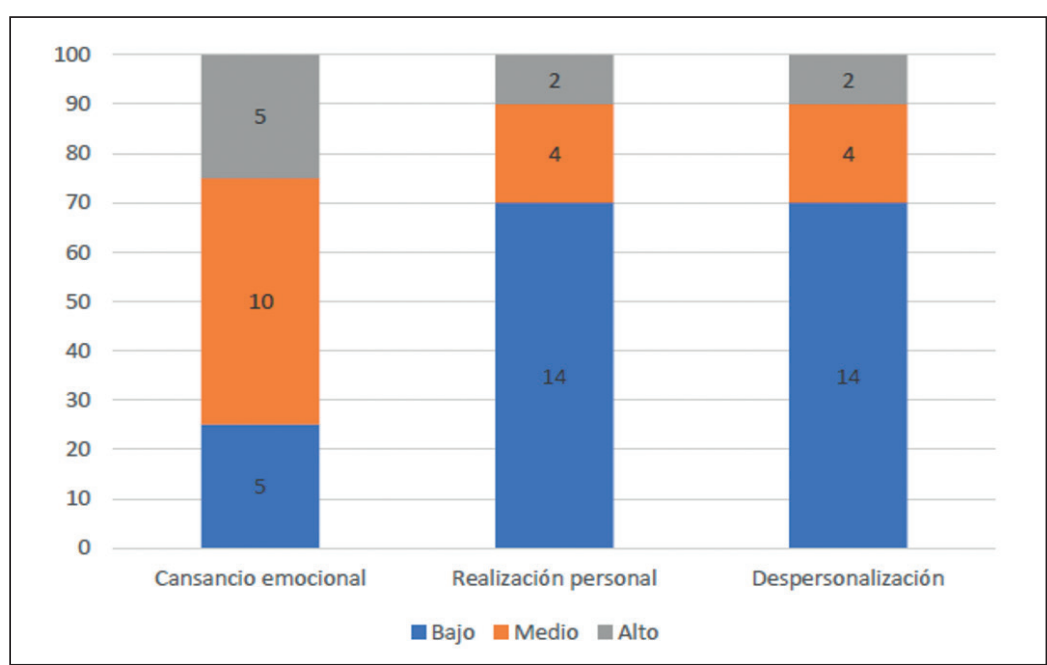

Figura 3. Número total de residentes y anestesiólogos que puntuaron en las categorías alto medio, bajo en cada subescala MBI $(n=20)$.

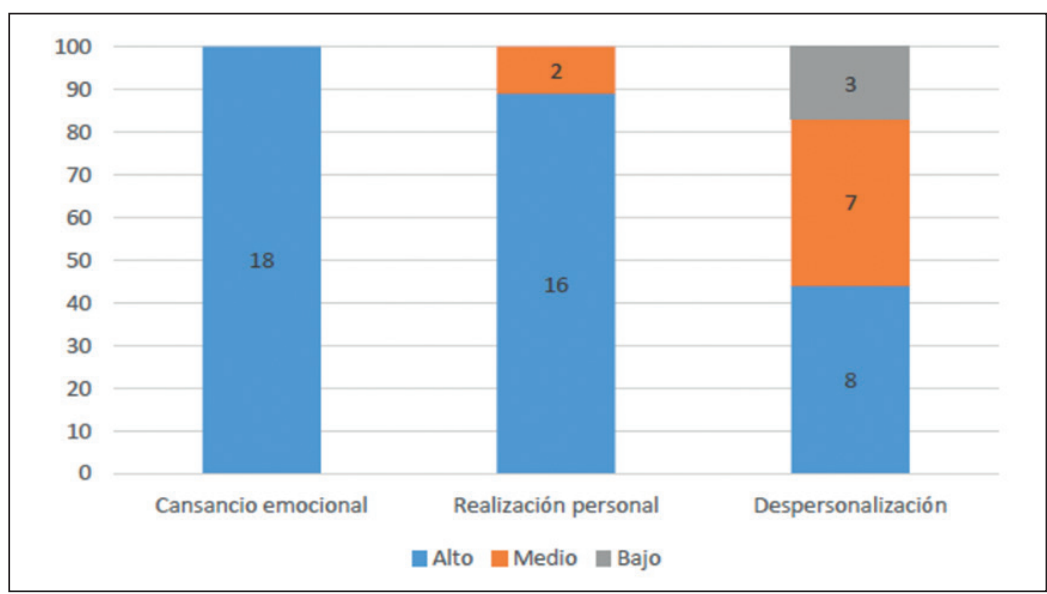

Figura 4. Número total de residentes de anestesiología y traumatología que puntuaron en las categorías alto, medio, bajo en cada subescala MBI $(n=18)$.

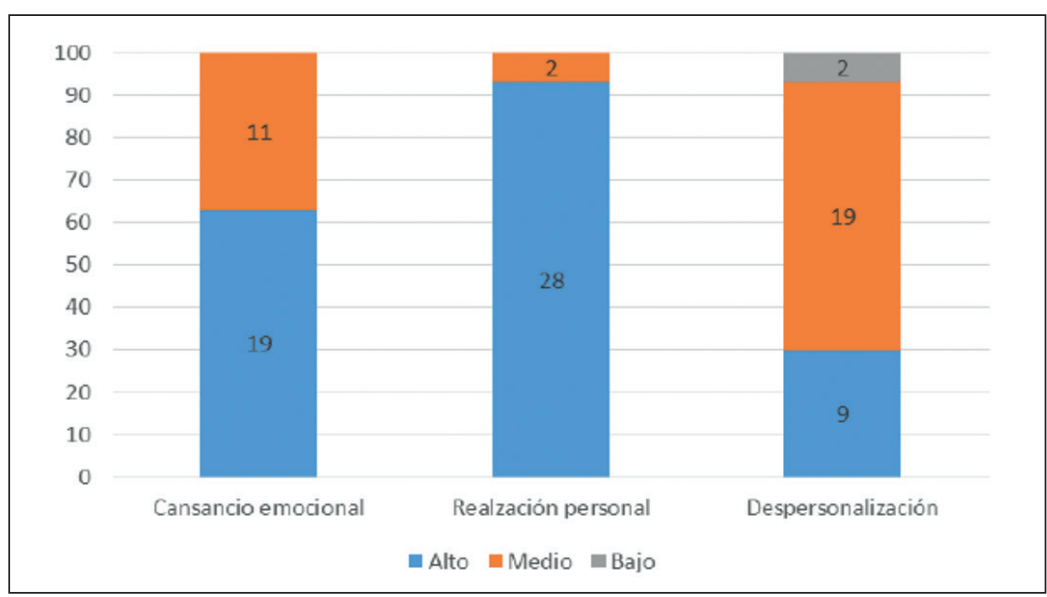

Figura 5. Número total de académicos-asistenciales de traumatología y anestesiología que puntuaron en las categorías alto, medio, bajo en cada subescala MBI $(n=30)$. 
Tabla 2. Tabla comparativa de variables género y horas de sueño según dimensión en el total de participantes

\begin{tabular}{|clcccccccc|}
\hline Dimensión & Variable & n & Promedio & SD & Variable & n & Promedio & SD & p \\
CE & Hombres & 33 & 29,24 & 6,0 & Mujer & 15 & 35,0 & 3,9 & $\mathbf{0 , 0 0 1}$ \\
RP & Hombres & 33 & 22,9 & 5,2 & Mujer & 15 & 23,9 & 5,0 & 0,53 \\
DE & Hombres & 33 & 12,7 & 3,4 & Mujer & 15 & 12,3 & 3,7 & 0,71 \\
CE & Duerme $+7 \mathrm{~h}$ & 9 & 27,22 & 7,22 & Duerme $-7 \mathrm{~h}$ & 39 & 31,94 & 5,5 & $\mathbf{0 , 0 3 3}$ \\
RP & Duerme $+7 \mathrm{~h}$ & 9 & 21,55 & 3,6 & Duerme $-7 \mathrm{~h}$ & 39 & 23,6 & 5,45 & 0,28 \\
DE & Duerme $+7 \mathrm{~h}$ & 9 & 12,66 & 2,4 & Duerme $-7 \mathrm{~h}$ & 39 & 12,6 & 3,7 & 0,96 \\
\hline
\end{tabular}

Tabla 3. Número de mujeres según niveles de puntuación $(n=15)$

\begin{tabular}{|lccc|}
\hline & Alto & Medio & Bajo \\
Cansancio emocional & $15(100 \%)$ & 0 & 0 \\
Realización personal & $13(86,6 \%)$ & $2(13,3 \%)$ & 0 \\
Despersonalización & $5(33,3)$ & $9(60 \%)$ & $1(6,6 \%)$ \\
Total & 33 & 11 & 3 \\
\hline
\end{tabular}

Tabla 4. Número de hombres según niveles de puntuación $(\mathbf{n}=33)$

\begin{tabular}{|lccc|}
\hline & Alto & Medio & Bajo \\
Cansancio emocional & $21(63,3 \%)$ & $12(36,36 \%)$ & 0 \\
Realización personal & $31(94 \%)$ & $2(6 \%)$ & 0 \\
Despersonalización & $12(36,3 \%)$ & $16(48,48 \%)$ & $5(15,15 \%)$ \\
Total & 64 & 30 & 5 \\
\hline
\end{tabular}

Tabla 5. Número de participantes que duermen más de 7 horas según niveles de puntuación $(n=15)$

\begin{tabular}{|lccc|}
\hline & Alto & Medio & Bajo \\
Cansancio emocional & $5(55,5 \%)$ & $4(44,44 \%)$ & 0 \\
Realización personal & $9(100 \%)$ & 0 & 0 \\
Despersonalización & $2(22,22 \%)$ & $7(77,77 \%)$ & 0 \\
Total & 16 & 11 & 0 \\
\hline
\end{tabular}

Tabla 6. Número de participantes que duermen menos de 7 h según niveles de puntuación $(n=39)$

\begin{tabular}{|lccc|}
\hline & Alto & Medio & Bajo \\
\hline Cansancio emocional & $31(79,48 \%)$ & $8(20,5 \%)$ & 0 \\
Realización personal & $35(89,7 \%)$ & $4(10,25 \%)$ & 0 \\
Despersonalización & $15(38,46 \%)$ & $18(46,15 \%)$ & $6(15,38 \%)$ \\
Total & 81 & 30 & 6 \\
\hline
\end{tabular}

La prevalencia en hombres fue del $100 \%$, la CE del 100\% (33 sujetos); una baja RP, el 100\% (33 sujetos), y la DE, el 84,8\% (28 sujetos). En la Tabla 4 se muestra la puntuación del total de hombres en las categorías alto, medio, bajo en cada subescala MBI.

La prevalencia del síndrome en aquellas personas que duermen más de $7 \mathrm{~h}$ de sueño fue del 100\%. Mientras que CE mostró una prevalencia del 100\% (9 sujetos); una baja RP el 100\% (9 sujetos) y DE el 100\% (9 sujetos). En la Tabla 5 se muestra la puntuación del total de sujetos que duermen más de $7 \mathrm{~h}$ en las categorías alto, medio, bajo en cada subescala MBI.

La prevalencia del síndrome en las personas que duermen menos de $7 \mathrm{~h}$ de sueño fue del 95,6\%. Mientras que CE mostró una prevalencia del 100\% (39 sujetos); una baja RP el 100\% (39 sujetos) y DE el 84,6\% (33 sujetos). En la Tabla 6 se muestra la puntuación del total de sujetos que duermen menos de $7 \mathrm{~h}$ en las categorías alto, medio, bajo en cada subescala MBI.

\section{Discusión}

La preocupación por el síndrome de burnout entre médicos residentes es por su asociación con depresión, ansiedad y abuso de sustancias psicoactivas. Hay reportes que muestran una prevalencia de $9,4 \%$ de pensamientos suicidas entre estudiantes de medicina de cuarto año e internos en Estados Unidos $^{13}$. Además de estar asociado a una mayor tasa de errores médicos y de interferir con la empatía y la comunicación con los pacientes y el equipo médico ${ }^{14}$.

Algunos autores apoyan la idea de que el síndrome de burnout se manifiesta por un incremento de los niveles de agotamiento emocional de los profesionales de la medicina ${ }^{4,15}$, pero existe controversia en términos del proceso secuencial en que aparece 
la sintomatología del síndrome. Golembiewsky y colaboradores $^{16}$, plantearon que el síntoma inicial es la despersonalización, posteriormente surge la baja realización personal en el trabajo y, finalmente, aparecen los sentimientos de cansancio emocional. Leiter y Maslach ${ }^{17}$ propusieron que el síntoma inicial serían los sentimientos de agotamiento emocional, posteriormente las actitudes de despersonalización $\mathrm{y}$, finalmente, la baja realización personal en el trabajo. También investigaciones han sugerido que el cansancio emocional incluye a otros aspectos del agotamiento: el cognitivo y el físico ${ }^{18}$, lo que no se evalúa en el MBI. Buunk y Schaufeli ${ }^{19}$, señalan que el síndrome tiene una doble etiología: por un lado, los procesos de intercambio social con los pacientes $\mathrm{y}$, por otro, los procesos de afiliación y comparación social con los compañeros. Gil-Monte, Peiró y Valcárcel añaden el aspecto organizacional en la etiología del síndrome y plantean la consideración de variables que estimen las disfunciones del rol y los procesos de intercambio social no solo entre los profesionales y las personas a las que atienden, sino entre aquellos y la organización ${ }^{20}$.

Comparando los resultados de nuestro estudio con otros que evaluaron la prevalencia del síndrome de burnout en otros profesionales médicos en otros lugares del mundo, en particular anestesiólogos y traumatólogos, encontramos en general cifras considerablemente más elevadas. Por ejemplo, en anestesiólogos en un hospital de Brasil ${ }^{21}$, se encontró una tasa global de $10,4 \%$, lo que contrasta con $45 \%$ encontrado. En un grupo de 69 traumatólogos en Arabia Saudita, por otro lado, se encontró una prevalencia de despersonalización de 59;4\% lo que contrasta con el $82 \%$ en nuestro estudio ${ }^{22}$.

Según sexo, las diferencias pueden deberse a las experiencias ocultas de lo femenino, que además del rol médico se agregan la valorización de ciertas tareas como crianza, cuidado y tareas domésticas ${ }^{23}$. En Chile aún se forman, legitiman y reproducen estas ideas con la consecuente saturación de roles ${ }^{24}$. Las diferencias en el cansancio emocional, siendo mayor para quienes duermen menos de $7 \mathrm{~h}$, podría explicarse en términos de que la comorbilidad entre ${ }^{26}$.

En Cumbre de Bienestar de los Residentes del año $2017^{13}$, se diseñó estudio para seleccionar un programa de herramientas para mejorar el bienestar dentro de los residentes de especialidad de emergencias. Estas herramientas incluyeron: trabajar el síndrome de segunda víctima (la experiencia y el impacto negativo que sufren los profesionales sanitarios ante un evento adverso sobre un paciente, así como su necesidad de apoyo), meditación en conciencia plena y psicología positiva. En particular, el entrenamiento en conciencia plena o mindfulness se ha asociado con una reducción de burnout y aumento de satisfacción en profesionales de la salud y con una mejora en los cuidados a los pacientes ${ }^{26}$. En un estudio reciente, la participación en un entrenamiento $\mathrm{CBCT}^{\circledR}$ (Cognitively-Based Compassion Training), ayudó no solo en su regulación emocional sino social ${ }^{27}$, hizo disminuir los niveles de depresión y soledad de estudiantes de medicina. Otra estrategia es la novedad del estudio de protectores de estrés laboral hospitalario ${ }^{28}$, que se refiere a los recursos ambientales que los propios profesionales sanitarios establecen en común como soporte ante el estrés. Visualizando esto, se desarrolló un instrumento de medida que evalúa protectores ambientales de estrés hospitalario llamado ENPROS (ENvironmental PRotectors against hOspital work Stress $)^{29}$.

\section{Conclusión}

Tanto residentes como académicos asistenciales de traumatología y anestesiología mostraron un elevado cansancio emocional que coexiste con una baja realización personal en el trabajo y alta despersonalización. Si bien el ámbito de evaluación del MBI es el emocional, la exclusión de aspectos afectivos, cognitivos y conductuales constituye un enfoque parcial que necesita ser complementado y revisado.

En Chile, las políticas de protección están principalmente enfocadas en una Política de Seguridad y Salud Ocupacional comprometida en sus actividades operacionales y con la gestión de los riesgos, pero no se observan políticas de protección al estrés y salud mental que involucren contención y apoyo a la labor terapéutica de los médicos, lo cual resulta insuficiente para abordar la complejidad que representa un contexto hospitalario en la Región de la Araucanía. La creación de organizaciones o entornos laborales que promuevan el bienestar de los trabajadores es un imperativo categórico para las organizaciones hospitalarias.

\section{Responsabilidades éticas}

Protección de personas y animales. Los autores declaran que para esta investigación no se han realizado experimentos en seres humanos ni en animales.

Confidencialidad de los datos. Los autores declaran que en este artículo no aparecen datos de pacientes. 
Financiamiento. Vicerrectoría de Investigación y Postgrado, Dirección de Investigación de la Universidad de La Frontera. DIUFRO N DI16-0047.

Contribución de los autores. HL: Diseño y ejecución del estudio, análisis de datos, discusión de resultados y redacción manuscrito. Supervisión y aportes ene todas las fases del estudio. PA: Diseño junto a HL, ejecución del estudio, búsqueda biblio- gráfica, recolección y análisis de datos, discusión de resultados, redacción del manuscrito y envío. SFC: Búsqueda bibliográfica, discusión de resultados, redacción de manuscrito. JJ: Recolección de datos, discusión de resultados, redacción del manuscrito. Todos los autores han leído y aprueban el manuscrito.

Conflictos de interés: no hay.

\section{Bibliografía}

1. Selye H. Stress and general adaptation syndrome. Br Med J. 1950;1(4667):138392. doi: 10.1136/bmj.1.4667.1383

2. Landa V, Mena L. Estrés laboral en personal clínico hospitalario. Ter psicológica. 2003;21:137-51.

3. Maslach C, Schaufeli WB, Leiter MP. Job Burnout. Annu Rev Psychol [Internet]. 2001;52(1):397-422. Available from: http:/www.annualreviews.org/ doi/10.1146/annurev.psych.52.1.397 [citado el 18 de abril de 2018].

4. Maslach C, Jackson S. The Measurement of Experienced Burnout. J Occup Behav [Internet]. 1981;2(April 1980):99-113. Available from: http://onlinelibrary.wiley. com/doi/10.1002/job.4030020205/full [citado el 18 de abril de 2018].

5. Dewe P, Leiter M, Cox T. Coping, health and organizations [Internet]. 2005. Available from: http://dl.mozh.org/up/ FFFF.pdf [citado el 18 de abril de 2018].

6. Amoafo E, Hanbali N, Patel A, Singh P. What are the significant factors associated with burnout in doctors?: Table 1. Occup Med (Chic Ill) [Internet]. 2015;65(2):11721. Available from: http://www.ncbi.nlm. nih.gov/pubmed/25324485 [citado el 13 de agosto de 2018].

7. Golkar A, Johansson E, Kasahara M, Osika W, Perski A, Savic I. The Influence of Work-Related Chronic Stress on the Regulation of Emotion and on Functional Connectivity in the Brain. PLOS ONE/ www.plosone.org. 2014;9(9):e104550.

8. Instituto Nacional de Estadísticas Chile. Estadísticas Demográficas y vitales. Región de La Araucanía. 2011.

9. Gobierno de Chile. Caracterización socioeconómica Nacional (Casen). 2013.

10. Barbosa FT, Eloi RJ, dos Santos LM, Leão BA, Lima FJC de, de Sousa-Rodrigues
CF. Correlação entre a carga horária semanal de trabalho com a síndrome de burnout entre os médicos anestesiologistas de Maceió-AL. Brazilian J Anesthesiol. 2017;67:115-21

11. Astudillo P, Losada H. Prevalencia de Síndrome de Burnout en un Centro de Cirugía Académico-Asistencial Público en Chile. Rev Chil Cir. 2018;70:117-26.

12. Díaz Bambula F, Gómez IC. Research on burnout from 2000 to 2010 in Latin America. Psicol desde el Caribe [Internet]. 2016;33(1):113-31. Available from: http:// rcientificas.uninorte.edu.co/index.php/ psicologia/article/viewFile/6442/8065 [citado el 30 de noviembre de 2018].

13. Chung A, Smart J, Zdradzinski M, Roth S, Gende A, Conroy K, et al. Educator Toolkits on Second Victim Syndrome, Mindfulness and Meditation, and Positive Psychology: The 2017 Resident Wellness Consensus Summit. West J Emerg Med. 2018;19:327-31

14. Faivre G, Kielwasser H, Bourgeois M, Panouilleres M, Loisel F, Obert L. Burnout syndrome in orthopaedic and trauma surgery residents in France: A nationwide survey. Orthop Traumatol Surg Res. 2018;104:1291-5.

15. Ng R, Chahine S, Lanting B, Howard J. Unpacking the Literature on Stress and Resiliency: A Narrative Review Focused on Learners in the Operating Room. J Surg Educ. 2019;76:343-53.

16. Taris T, Le Blanc P, Schaufeli W, Schreurs P. Are there causal relationships between the dimensions of the Maslach Burnout Inventory?: A review and two longitudinal test. Work Stress 2005;19:238-55

17. Leiter M, Maslach C. The Impact of Interpersonal Environment on Burnout and Organizational Commitment. J Organ BEHAOVIOR 1988;9:297-308.
18. Halbesleben JRB, Demerouti E. The construct validity of an alternative measure of burnout: Investigating the English translation of the Oldenburg Burnout Inventory. Work Stress 2005;19:208-20.

19. Buunk BP, Zurriaga R, Peíró JM, Nauta A, Gosalvez I. Social Comparisons at Work as Related to a Cooperative Social Climate and to Individual Differences in Social Comparison Orientation. Appl Psychol AN Int Rev [Internet]. 2005;54(1):61-80 Available from: https://s3.amazonaws. com/academia.edu.documents/39931768/ Social_Comparisons_at_Work_as Related to20151112-4371-1tkqu16. pdf?AWSAccessKeyId=AKIAIWO WYYGZ2Y53UL3A\&Expires $=152$ 4079711\&Signature $=$ oEYkHwRry6 4GbGoAKCrnXkpixFQ\%3D\&respo nse-content-disposition $=$ inline $\% 3 \mathrm{~B}$ filename\%3DSocial_Comparisons_at Work_as_Related_to.pdf [citado el $18 \mathrm{de}$ abril de 2018].

20. Gil-Monte P. Some reasons to Consider psicosocial Risk in the Work and its consequences on Public Health. Rev Esp Salud Publica 2009;83:169-73.

21. Magalhães E, Oliveira ÁCM de $\mathrm{S}$, Govêia CS, Ladeira LCA, Queiroz DM, Vieira CV. Prevalência de síndrome de burnout entre os anestesiologistas do Distrito Federal. Brazilian J Anesthesiol. 2015;65:104-10

22. Sadat-Ali M, Al-Habdan IM, AlDakheel DA, Shriyan D. Are orthopedic surgeons prone to burnout? Saudi Med J. 2005;26:1180-2.

23. Arcos GE, Molina VI, Fecci PE, Zúñiga AY, Márquez MM, Ramírez M, et al. Descubriendo el género en el currículo explícito (Currículo formal) de la educación de tercer ciclo, Universidad 
Austral de Chile 2003-2004. Estudios pedagógicos. 2006;32:33-47.

24. Abramo L, Valenzuela ME, Pollack M. Equidad de género en el mundo del trabajo en América Latina. Avances y desafíos cinco años después de Beijing [Internet]. Ginebra; 2000. Available from: http://white.lim.ilo.org/spanish/260ameri/ publ/docutrab/dt-130/texto_completo.pdf [citado el 21 de marzo de 2019].

25. Medrano-Martínez P, Ramos-Platón MJ. [Cognitive and emotional alterations in chronic insomnia]. Rev Neurol. [Internet]. 2016;62(4):170-8. Available from: http:// www.ncbi.nlm.nih.gov/pubmed/26860722 [citado el 5 de marzo de 2020].

26. Goodman M, Schorling J. A mindfulness course decreases burnout and improves well-being among healthcare providers. Int J Psychiatry Med [Internet]. 2012;43(2):119-28. Available from: http:// journals.sagepub.com/doi/abs/10.2190/ PM.43.2.b [citado el 30 de noviembre de 2018].

27. Mascaro JS, Kelley S, Darcher A, Negi LT, Worthman C, Miller A, et al. Meditation buffers medical student compassion from the deleterious effects of depression. J Posit Psychol. 2018;13:13342.

28. Astudillo Díaz PR, Ma Alarcón Muñoz A, Lema García ML. Protectores de estrés laboral: percepción del personal de enfermería y médicos, Temuco, Chile. Available from: https://scielo.conicyt.cl/ pdf/cienf/v15n3/art_12.pdf [citado el 13 de abril de 2018].

29. Astudillo P, Losada H, Burgos M. Hacia la construcción de un modelo de medida de protectores de estrés en el entorno laboral hospitalario. DIUFRO N ${ }^{\circ}$ DI16-0047. Temuco; 2017. 